\title{
Three-dimensional cartilage-derived construct derived from multipotent mesenchymal stromal cells
}

\author{
Oleg Makeyev ${ }^{1,2}$, Arteom Korotkov $^{1,2}$, Svetlana Kostyukova ${ }^{1,2}$, Daria Sichkar ${ }^{1,2}$, and \\ Elizaveta Yakovleva ${ }^{1}$ \\ ${ }^{1}$ Ural State Medical University, 620028, Repin Str., 3, Ekaterinburg, Russian Federation \\ ${ }^{2}$ Institute of Medical Cell Technology, 620026, Ekaterinburg, Russian Federation \\ ${ }^{3}$ Ural Federal University, 620002, Mira Str., 19, Ekaterinburg, Russian Federation
}

\begin{abstract}
Damage to the cartilage surface of the joints is a common pathology. Conventional drug therapies are effective only at the initial stage of the disease and only slow down the further development of the disease. In subsequent stages, it is necessary to apply surgical methods from replacing a defect to complete prosthetics of a damaged joint.

In order to replace defects in cartilage tissue, a three-dimensional bioequivalent was created based on autologous multipotent mesenchymal stromal cells.
\end{abstract}

\section{Introduction}

Osteoarthrosis (synonyms: deforming osteoarthrosis, arthrosis, deforming arthrosis) is a degenerative joint disease caused by damage to the cartilage of the articular surfaces.

A large-scale study conducted in Russia revealed manifest (accompanied by clinical symptoms) osteoarthritis in $64.3 \%$ of those examined over 15 years of age) [1].

The first place in pharmacotherapy is occupied by non-steroidal anti-inflammatory drugs prescribed to relieve pain and inflammation. In the phase of acute pain, when osteoarthritis can be complicated by synovitis, intra-articular administration of corticosteroids (diprospan, triamcinolone, and hydrocortisone) is used to relieve pain and inflammation [2]. Chondroprotectors (chondroitin sulfate and glucosamine) are still used as a course of treatment orally, intramuscularly, intraarticular in the first and second stages of the disease. Hyaluronic acid preparations are also used for intraarticular administration. However, the results of modern scientific studies show the lack of effect of these drugs compared with placebo [3]. A network meta-analysis of 10 major clinical trials conducted in 3803 patients with OA of the knee and hip joints failed to reveal any clinically significant effect of glucosamines, chondroitin sulfates or a combination of these in reducing joint pain or slowing joint space narrowing compared to placebo [4]. Physiotherapeutic treatments have not shown significant clinical efficacy.

In the presence of indications for surgery, the method of choice may be the joint replacement. However, in a whole series of cases, an endoprosthesis based on various materials (metals, sprayed metals, etc.) provides only a temporary effect. 
Thus, the search for new methods of treatment of osteoarthritis is an urgent task. Progressive osteoarthritis is a pathology for the treatment of which currently there are no effective methods. Successful attempts to use cell technology are known. Meanwhile, the effectiveness of this approach remains ambiguous.

Currently, one of the promising methods is the implantation of autologous tissueengineering structures, which involves the creation of a three-dimensional construct (scaffold), which is formed in vitro, followed by implantation into the lesion of the articular cartilage. Three components are used to construct the scaffold: matrix, cells, and cytokines of directed chondrogenesis.

In this case, the matrix is a complex with desired properties, providing mechanical structural strength and three-dimensional orientation for the applied cell culture, which allows a specific chondroid cell phenotype to be maintained for a long time. The matrix can be made of synthetic or organic materials and should have properties such as biocompatibility, resorbability, nontoxicity, low immunogenicity, support for chondrogenesis, and homogeneous distribution of cells over the material of the carrier.

After transplantation, cells synthesize a matrix that replaces the scaffold material as it is resorbed. The source of the cells may be chondroblasts of their own cartilage tissue or progenitor cells of varying degrees of maturity.

Cultivation and stimulation of the chondrocytic culture integrated into the matrix is carried out in special devices - bioreactors, which allow you to control the physical and chemical parameters that determine the differentiation of the formed transplant.

Currently, the implantation of chondrocytes under the membrane or on the matrix allows you to close defects from 1 to $7 \mathrm{~cm}^{2}$.

The scientific approach involves the use of chondroblasts to restore joint function. Chondroblasts is performed by a tissue-engineering design based on autologous chondroblasts and a biodegradable carrier.

After transplantation, the cells that form the basis of the tissue engineering construct synthesize a matrix that replaces the carrier material as it is resorbed.

The methodological approach involves obtaining a patient's tissue sample $\left(2-6 \mathrm{~mm}^{3}\right)$, isolating the primary cell culture and increasing the number of cells to the required volume (determined by the area of the lesion).

Based on the foregoing, the purpose of this study is to develop a technology for creating a three-dimensional tissue-engineering design based on human chondroblasts.

The objectives of this study were:

- to develop a reproducible protocol for producing chondroblasts by directed differentiation of multipotent mesenchymal stromal cells suitable for creating a 3D tissueengineering construct;

- to study the cellular composition of a fabricated tissue-engineering structure.

\section{Materials and methods}

We used MMSC isolated from adipose tissue of the anterior abdominal wall of a person obtained by liposuction in a hardware manner based on the informed consent of patients. The isolation of MMSC was carried out by a mechano-enzymatic method using collagenase as a dispersant (BioloT, St. Petersburg). Adipose tissue was mixed with collagenase in a ratio of 1: 2 by volume and incubated at $37^{\circ} \mathrm{C}$ with constant stirring for 2 hours. After fermentation, the resulting suspension of cells was passed through a nylon filter and washed twice from the dispersant solution with a nutrient medium.

The viability of the obtained cells was evaluated by trypan blue staining..

In order to increase cell mass, MMSC was cultured using DMEM culture medium (Sigma-Aldrich,) supplemented with $10 \%$ fetal bovine serum (Hy-clone). For 
differentiation in the chondrogenic direction, a combination of insulin, transforming growth factor and ascorbate was introduced into the culture medium [5]. Upon reaching the monolayer, cells from the surface of the culture vial were removed with a trypsin-Versen solution at concentrations of $0.25 \%$ and $0.2 \%$, respectively.

Vital morphology of cell cultures and their photo-documentation was performed using an Olympus CKX 41 inverted microscope.

The cryopreservation of the obtained MMSC cell mass was performed by the stepwise method: gradual cooling to $-84^{\circ} \mathrm{C}$ in a Mr. container Frosty (Nanc), followed by immersion in liquid nitrogen. DMSO (MP Biomedicals) at a concentration of $10 \%$ was used as a cryoprotectant.

Fabric fabrication was carried out by the author's method with cultivation staging, which allows to give the design a given shape and structure. The latter involved modeling a structure consisting of layers with varying degrees of cell maturity - more mature and differentiated - in the center, young cells - on the periphery.

After cultivation, the resulting construct was fixed in a standard manner, after which sections were prepared and stained according to Mallory, Weigert-Van Gieson and Alcian blue $\mathrm{pH} 2.5$.

Fluorescence microscopy (qualitative analysis) and flow cytofluorimetry (quantitative analysis) were used to evaluate the expression of specific positive and negative markers. Requirements for the expression of receptors on the surface of the studied cells: for CD73, CD90, CD105 - at least $95 \%$ of the cells in the sample, for CD31, CD1, and CD14 - no more than $2 \%$ of the cells.

In order to assess cell polypotency, special media containing growth and differentiation factors in chondrogenic and osteogenic directions were used. To confirm the differentiation of cultures, biochemical markers were analyzed (alkaline phosphatase, type I collagen, osteopontin, osteonectin, osteocalcin, bone sialoprotein, type II and IV collagen, sulfated proteoglycans, as well as a number of membrane surface markers determined by immunofluorescence methods: RunX-1 BMP-2, BMP-4, PTH receptor).

The data obtained were processed by nonparametric statistics. The statistical difference between the groups is established using the Kruskal-Wallis method with further processing by the method of multiple comparisons according to Dunn. The significance of differences compared to baseline is determined using the Wilcoxon test for related samples.

\section{Results and Discussion}

Identification of MMSC derived from human adipose tissue

Cells obtained from human anterior abdominal wall fat showed high adhesion on culture plastics. Even at the isolation stage, the adhesion property was used as a sort of sorting method: only those cells that were able to fix on plastic during the first days of cultivation were preserved in the culture, the rest were removed during the first or subsequent passages.

Adhesive cells had a spindle-shaped or process (fibroblast-like) shape. During proliferation, the cells showed the ability to locomotion on the cultural surface, gradually spreading to the maximum possible area with the loss of contact with each other.

As the culture approached the state of the monolayer, cell proliferation slowed noticeably, and then stopped. Thus, the culture demonstrated the ability to contact inhibition, which refers to one of the manifestations of feelings of their own pool. This characteristic is typical of all cells that completely or partially differ in the properties of "stemming" and / or that realize restoration of the cell population.

The removal of cell culture from the surface by trypsinization was accompanied by a change in the shape of the cells from the process to spherical, which is associated with cell 
adhesion. Trypsin, destroying surface adhesive proteins, deprived the cell of the basis of fixation on the surface, after which the cells acquired a spherical shape and were washed off from the surface of the culture plastic.

After neutralizing trypsin and transferring the removed culture to a new surface, after 30-40 minutes the cells were firmly fixed on the plastic, ceased to give in to the usual mechanical washing off and gradually acquired a process form.

Thus, we can conclude that the fixation of cells on the culture plastic and their process shape indicates the ability to adhere - one of the main distinguishing features of MMSC, and the trypsinization effect - to the adhesion mechanism realized by trypsin-labile surface proteins.

The second necessary identification criterion for MMSC is the expression of a set of specific surface markers. In accordance with the accepted criteria, MMSCs should express CD73, CD90, CD105 markers and differ in the minimal expression of CD34, CD45, CD14, CD19 (and some other negative markers).

The method of fluorescence microscopy of cells isolated from fat of the anterior abdominal wall, demonstrated the expression of the necessary set of positive markers (CD73, CD90, CD105). Despite the fact that the technique is not quantitative, it leaves the possibility only for a qualitative determination, however, the fluorescence intensity suggested a sufficiently strong expression of the studied markers.

According to the definition of CD14, CD34 and CD45, fluorescence microscopy did not actually allow us to identify the fluorescence of colors corresponding to the fluorochromes used, which indicates an extremely low level of expression of negative markers.

Quantitative analysis of markers using flow cytofluorimetry showed unidirectional results with fluorescence microscopy. The average expression level of the CD73, CD90, and CD105 markers was higher than 90\% (92.35 to 95.19) (CytoFLEX TM).

It seems that the MMSC culture under study contains a small fraction of cells that do not express positive markers. However, the proportion of these cells does not quantitatively exceed $4-7 \%$, which suggests that most of the cells in the studied samples belong to MMSC and the culture parameters as a whole correspond to the MMSC properties.

In favor of the aforesaid, the expression of negative markers CD34, CD45, and CD14 is indicated, the analysis of which revealed no differences from the indicators of negative control (less than 1\%). This indicator fully complies with the requirements for pure MMSC cultures (expression of negative markers no more than $2 \%$ ) (Figure 1).
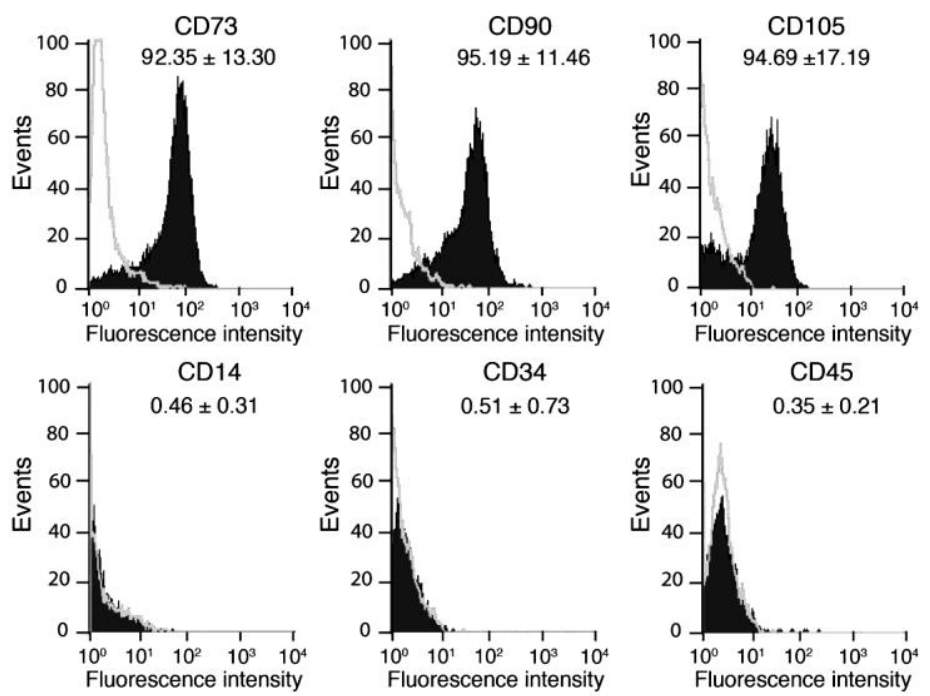

Fig. 1. Quantitative analysis of CD markers 
A standard analysis of signals received from intact cells by laser beam scattering showed that the sample contained at least $95 \%$ of intact cells, which is sufficient to verify the results as reliable.

The third necessary identification criterion for MMSC is the ability to differentiate in three directions: osteogenic, chondrogenic and adipogenic.

For chondrogenic differentiation, the MMSC culture was reseeded with medium density onto the culture surface. After "fixing" on the surface (within 2 days), the standard medium was changed to a special chondrogenic, after which the cells were cultured for 10 days.

Already on the 4th day of cultivation in the medium, changes in cell morphology became noticeable. The process and spindle-shaped (fibroblast-like) form changed to a more rounded, and the nuclear-cytoplasmic ratio also increased (this, as a rule, indicates an increase in synthetic activity). It was noted that the cells almost lost their mobility, and the average distance between the cells decreased.

From day 5, a marked increase in the mass of intercellular substance was observed. When fixing the resulting culture, the latter acquired the form of a richly white coating. When trypsinized, cells were detached layer by layer from plastic, remaining firmly adhered to each other in the form of a monolayer. This observation is explained by the appearance of a large mass of intercellular substance, which maintains strong contacts after a short trypsin treatment, which is characteristic of cartilage tissue. For reliable determination of cell differentiation, histological dyes were used.

Weingert-Van Gieson staining (Figure 2) allowed us to determine that the intercellular matrix contains a large amount of collagen. It should be noted that the matrix in this case did not have a pronounced fibrillar structure; on the preparations, it appeared as raspberrycolored fields (of varying degrees of saturation depending on the degree of collagen maturity). Light brown elastic fibers that accompanied collagen structures were also determined on the preparations. Non-fibrillar masses of collagen, the presence of a small amount of elastic fibers, a large amount of intercellular substance allows us to characterize the emerging cartilage tissue.

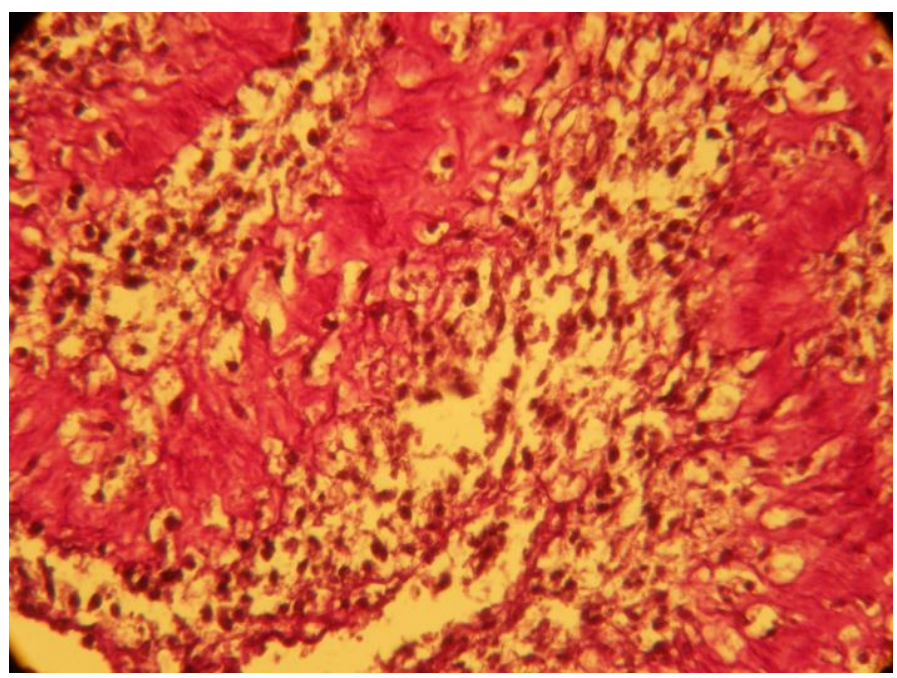

Fig. 2. Weigert-Van Gieson stain, 100x magnification

Staining with Alcian blue (at $\mathrm{pH}$ 2.5) revealed another sign of the extracellular matrix characteristic of cartilage - the presence of acid mucopolysaccharides (Figure 3). On stained preparations, they are presented in the form of homogeneous fields with various shades of blue. The absence of pronounced fibrillarity in the structure of the matrix is noted. 


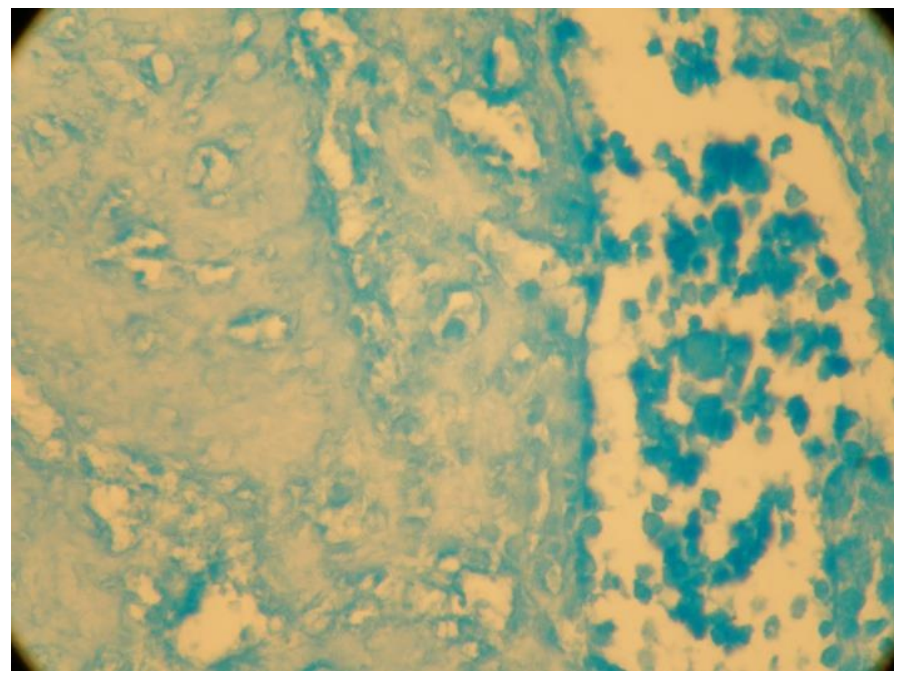

Fig. 3. Staining with Alcian blue $\mathrm{pH} 2.5 ; 100 x$ magnification

Interesting data were obtained using Mallory stain (Figure 4). These preparations revealed pronounced collagen structures in the intercellular matrix. In this case, areas with a fibrillar structure were observed.

In some areas of the preparation, collagen (colored blue) was present in the form of separate fragments, and the main volume was occupied by the cells themselves (visible by nuclei painted in raspberry color). In other areas, the culture showed signs of mature cartilage: a large amount of collagen stained blue; sparseness of cells; pronounced fields of a dense intercellular matrix with intermittent groups - are characteristic morphological features of chondrogenic differentiation.

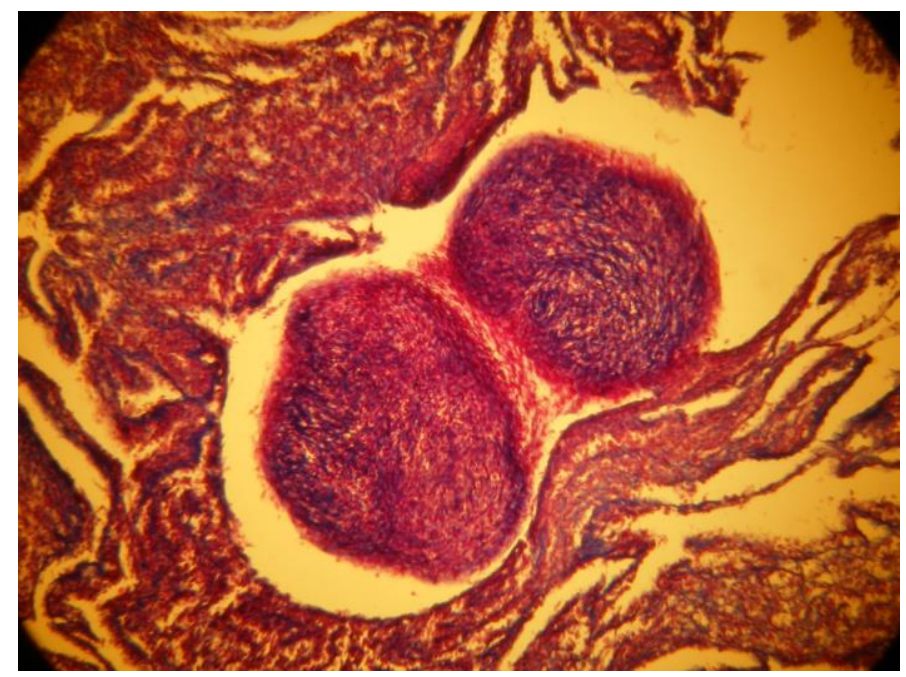

Fig. 4. Mallory stain; 800x magnification

Immunohistochemical studies allowed us to confirm the results obtained using histological methods. On preparations in the intercellular matrix revealed collagen type 2 (colored brown) in large quantities. 
Thus, the identification of the structural components of cartilage tissue in variously colored preparations, as well as its specific features, convincingly prove the differentiation of MMSC in the chondrogenic direction.

Another direction of MMSC differentiation (from those directions that are necessary for identifying a culture) is osteogenic.

After replacing the usual culture medium with a medium with osteogenic potencies, visible morphological changes in the culture were observed by the 6 th day. These changes have common features with the process of chondrogenic differentiation and are associated with the synthesis of a large amount of intercellular substance. The monolayer of cells also gained density, poorly resuspended during trypsin treatment. However, these signs were less pronounced than in a culture with chondrogenic differentiation (a smaller volume and a lower degree of density of the intercellular matrix). At the same time, the cells were not rounded, retained the process shape, and the processes became more pronounced. The cells retained the ability to move along the cultural surface, moved from each other, leaving a distance between themselves.

The morphological properties of the resulting culture, although reminiscent of the culture obtained during chondrogenic differentiation, had significant differences, indicating that in this case we received a different differon. The differences are clearly visible on the Mallory-stained preparation, on which collagen fibers are present in the matrix, but do not constitute its main part, but have a much more pronounced fibrillar structure (Figure 5). A less dense arrangement of cells is noted.

To identify the osteogenic direction of differentiation, the method of staining with alizarin red was used. This method is considered the most highly specific in relation to bone tissue and is considered as a method used to identify the osteogenic direction of MMSC differentiation. Analysis of the drug revealed areas of emerging bone tissue (mineralized matrix) that form after a week of cultivation. By the end of 3 weeks of cultivation, the matrix occupied about $70 \%$ of the culture surface.

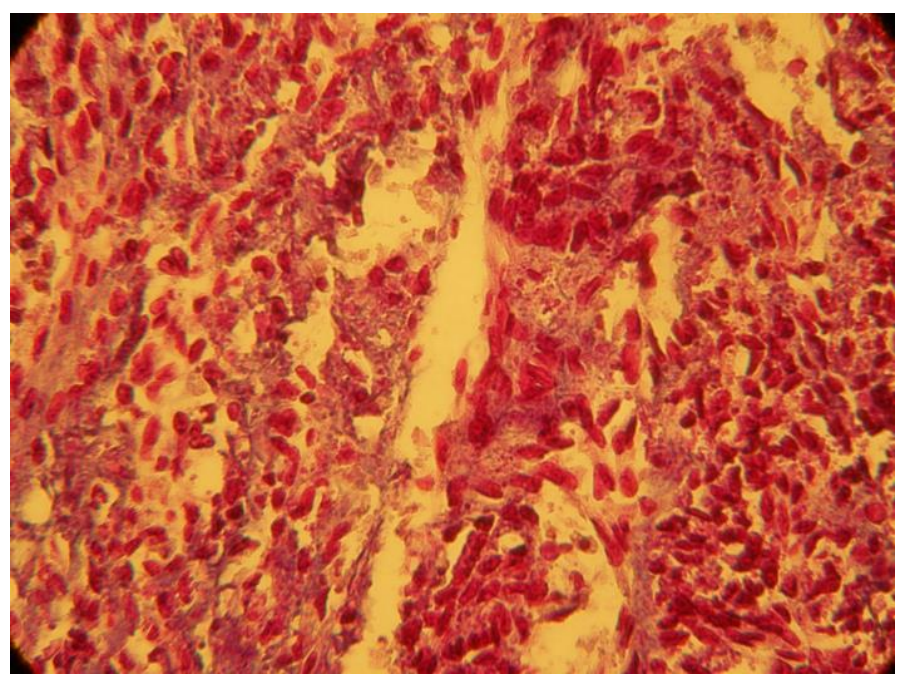

Fig. 5. An emerging bone tissue. Mallory stain, 200x magnification

Thus, the fact of osteogenic differentiation is histologically confirmed.

The third direction of cell differentiation, used as evidence of the belonging of the culture to MMSC, is adipogenic. Differentiation in this direction was carried out spontaneously, when cultured in a standard medium for more than 6 weeks. Single adipocyte-like cells appeared in the culture, which were clearly distinguishable by phase- 
contrast microscopy. Adipocyte-like cells had a nearly spherical shape, highly vacuolated cytoplasm containing lipid inclusions. The inclusion of inclusions in lipids was established by staining the contents of vacuoles with dye Azur Eosin (Figure 6).

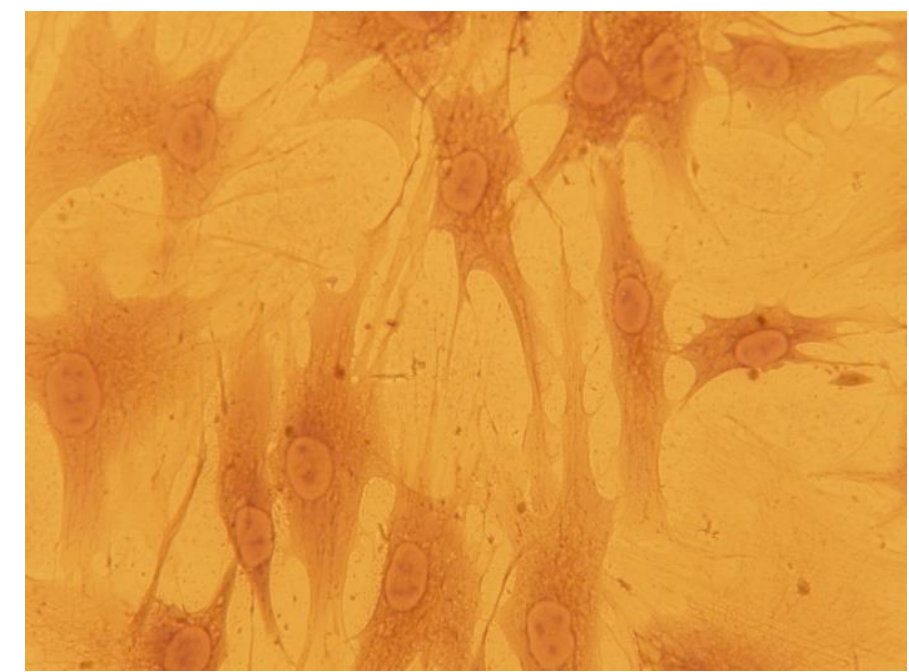

Fig. 6. Lipid inclusions in cells. Coloring Azur Eosin, 400x magnification

Thus, the studies established the ability of the obtained cell culture to adhere to the surface of the culture plastic; expression of three positive (CD73, CD90, CD105) and three negative markers, the possibility of differentiation in orthodox directions (chondrogenic, osteogenic, adipogenic) has been proved, which allows us to identify the studied cell cultures as MMSC cultures.

Morphological characteristics of the three-dimensional tissue-engineering cartilage design

Research on the development of a technology for producing a three-dimensional tissueengineering structure (scaffold), which can be implanted in the area of damage to the articular cartilage, allowed us to form a cartilage structure corresponding to a given disk shape $2.5-3 \mathrm{~mm}$ in height and $19 \mathrm{~mm}$ in diameter. It has a soft texture along the periphery, an elastic center and an uneven pink-yellow color (Figure 7).

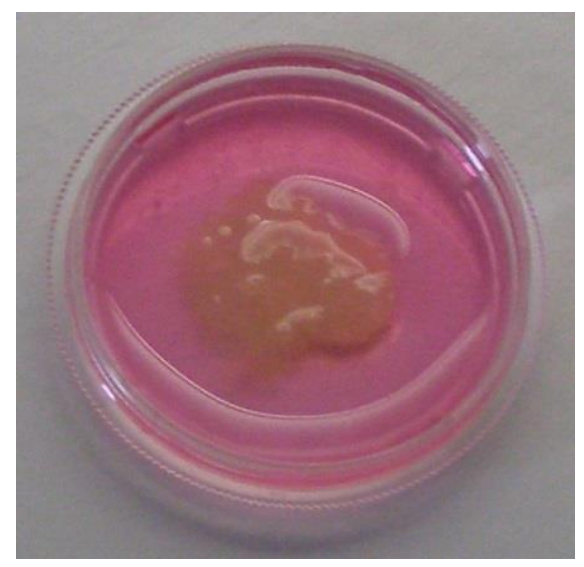

Fig. 7. The resulting cartilage construction 
Microscopically, the tissue-engineering construct contains round, oval or triangular cells. More common are single-core, sometimes dual- or multi-core cells. The cell nuclei of the central part of the structure are large and, as a rule, have an eccentric arrangement.

The cartilage cells in the created design are at different stages of differentiation: the precursors of chondroblasts and young chondroblasts, with an outgrown shape and small size, are located on the periphery. In the central part of the cell, they are more differentiated, have a larger, ovoid shape and are compactly arranged in 2-4 cells, forming the so-called isogenic groups that arise after the last division, with a lacunar structure. The gradation of the degree of differentiation from the periphery to the center, in general, resembles the structure of the cartilage tissue that forms in vivo. The latter indicates the possibility of repeating the in vitro structure of cartilage tissue by tissue-engineering methods.

A characteristic feature of the created design is the presence of a large amount of intercellular substance, including fibrous structures and amorphous substance. The main part of the fibrous structures is represented by collagen, which is a type of primary fibril and is the main element of the fibrous formations of cartilage. The latter are especially noticeable when stained according to Mallory. Another type of fiber, which is also contained in the matrix of the obtained structure, is elastic fibers accompanying collagen and visualized during Weigert-Van Gieson dyeing (Figure 8).

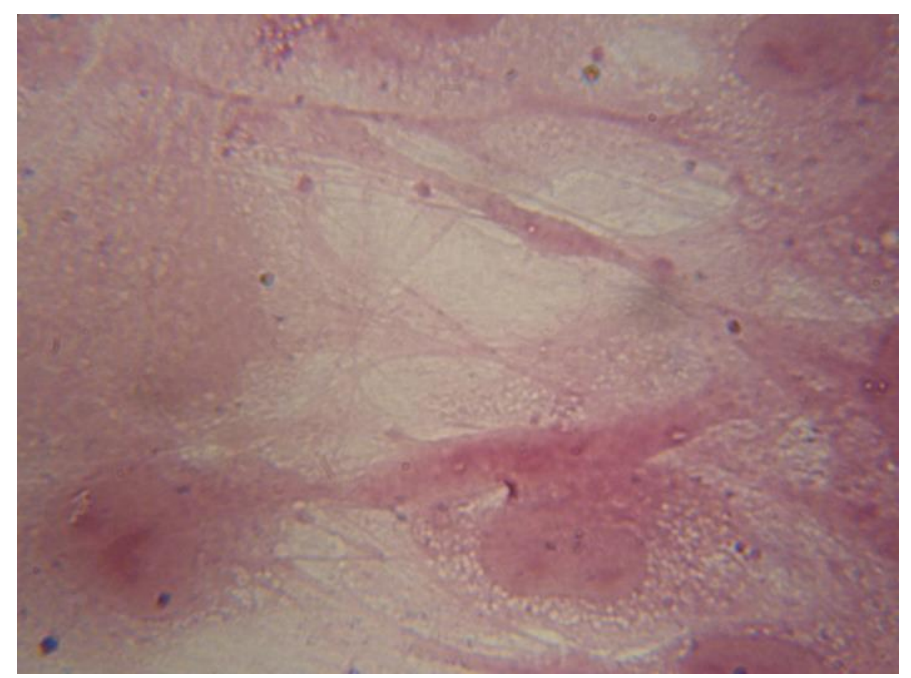

Fig. 8. Collagen structures Coloring according to Weigert-Van Gieson, magnification 800x

The main structural substance contains acid mucopolysaccharides. In terms of the stainability of the main dye (Mallory), the main substance differs from the oxyphilically stained collagen and is well visualized when painted with acidic dyes (alcian blue). In the transition zone from more mature to less mature cartilage, signs of metachromasia are observed. The correlation in the design of isogenic cell groups and fibrous formations in interterritorial areas indicates the greatest strength of the simulated tissue in the central part of the structure, which corresponds to the functional features of the cartilage tissue that forms in the body.

The design created allows us to observe the stages of cartilage histogenesis, in particular in the region of transition of young cartilage to mature, the change in oxyphilicity (stage 1 of the development of cartilage tissue) to basophilicity of the main substance (stage 2) with a return to oxyphilicity (stage 3 ). The last stage coincides with the time of the appearance of fibrils (Figure 9). 


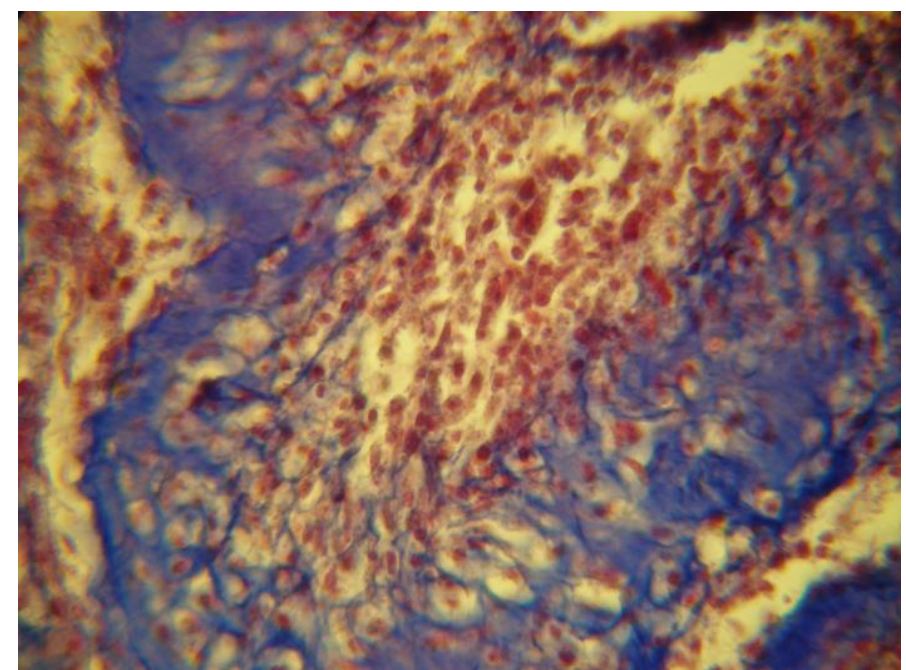

Fig. 9.Mallory stain, 100x magnification. A section of cells with chondro differentiation

The important question is whether the created structure belongs to one or another type of cartilage tissue - hyaline, elastic or fibrous. Apparently, the formed structure in its morphological structure is closer to fibrous, since elastic fibers are quantitatively smaller than collagen fibers, they do not branch and are oriented in the same direction as collagen fibers.

It is known that blood vessels practically do not occur in mature cartilage tissue, and nutrition occurs diffusely. This is a fundamental point in the practical use of such a design, since the main problem of any three-dimensional and extended tissue-engineering transplants is the lack of an adequate vascular network and, as a result, causes central necrosis. Cartilage cells and constructions based on them are devoid of this drawback, since in natural conditions tissue is fed due to marginal zones through the perichondrium. This suggests a high survival rate of such a transplant and a positive clinical result.

\section{Conclusion}

As a result of studies from cells of multipotent mesenchymal cells, cells showing phenotypic signs of chondroblasts were obtained.

Using the cells obtained in this way, a three-dimensional structure of cartilage is formed. Moreover, the spatial interdependence of cells and intercellular substance in a three-dimensional structure corresponds to that observed in native cartilage

\section{References}

1. N. Korshunov, V. Marasaev, E. Baranova, N. Parusova, L. Lutskova, RMZh., 23, 1320 (2003)

2. W. Zhang, W.B. Robertson, J. Zhao, W. Chen, J. Xu, Front Endocrinol (Lausanne), 10, 431 (2019)

3. X. Zhu, D. Wu, L. Sang, Y. Wang, Y. Shen, X. Zhuang, M. Chu, L. Jiang, Clin. Exp. Rheumatol, 36 (2018)

4. H.S. Vasiliadis, K. Tsikopoulos, World J. Orthop., 8, 1 (2017) 
5. P.A. Zuk, M. Zhu., P. Ashjian, D.A. De Ugarte, J.I. Huang, H. Mizuno, Z.C. Alfonso, J.K. Fraser, P. Benhaim, Mol. Biol. of the Cell., 13, 4279 (2002) 